\title{
One and Two Dimensional Arrays of Membrane Proteins Stabilized by Amphipol
}

\author{
Wanatchaporn Arunmanee ${ }^{1}$, J. Robin Harris ${ }^{1,2}$ and Jeremy H Lakey ${ }^{1}$ \\ 1. Institute for Cell and Molecular Biosciences, Newcastle University, Framlington Place, Newcastle \\ upon Tyne NE2 4HH, United Kingdom \\ 2. Institute of Zoology, University of Mainz, D-55099 Mainz, Germany
}

\begin{abstract}
All living cells are sounded by a lipid bilayer which forms the cell membrane and separates the aqueous intra- and extra cellular compartments. The lipid bilayer itself creates a five nanometer thick environment which is hydrophobic at its center and hydrophilic where it meets the bounding environments (Fig1). As throughout the rest of the cell, the membrane contains a repertoire of proteins which confer on it active functions such as transport, metabolism or molecular recognition. However, unlike the water soluble proteins found in the cytoplasm, the membrane proteins are highly hydrophobic where they are buried in the bilayer but hydrophilic where they face the compartments on either side. Because of this the study of purified membrane proteins is complicated by the need to recreate the mixed environment of the membrane. In some cases membranes containing only one type of protein are found in nature and one of these, the purple membrane from photosynthetic bacteria, was used to obtain the first molecular structure of a membrane protein using electron microscopy by Henderson and Unwin [1].

In most cases the membranes contain low levels of the required protein in a mixture with others and thus the protein needs to be extracted from the original membrane and purified before it can be studied in a reductionist way. To extract the protein from the membrane workers generally use detergents which dissolve the membrane and from a protective micelle around the hydrophobic regions of the membrane protein (Fig.1). Whilst the basis of many successful studies, detergents have negative aspects and, in particular, they need to be present at a concentration above that needed to from micelles. Under these conditions a complex mixture exists of protein containing micelles, empty micelles and free detergent. Furthermore the micelle is dynamic and does not recreate the surface pressure of the bilayer and can be destabilizing.
\end{abstract}

To provide an alternative technology to overcome this problem, Popot and colleagues developed a novel class of detergent called Amphipol (APol), an amphipathic polymer comprising a hydrophilic backbone randomly grafted with hydrophobic chains. This structure enables APol to stabilize MP in detergent-free aqueous solutions $[2,3]$.As APol forms multiple contacts with hydrophobic surface of MP (Fig1), the rate of dissociation of APol from proteins is slow [3] Theoretically, APol can solubilise membrane proteins in a near absence of free APol $[2,3]$. Since its introduction almost 20 years ago structural studies on several MP stabilized by APol have been carried out using electron microscopy (EM) [4-10]

Here we show that the removal of free amphipol from purified preparations of the bacterial outer membrane protein OmpF results in the formation of linear arrays of individual protein trimers (Fig1). These filaments have been imaged by electron microscopy using negative staining [11] and reveal that the proteins lie on their hydrophobic face on the carbon grid. This appears to show that there is a preferred intermolecular interface which promotes the use of long linear arrangements of the protein. This regular arrangement may provide the basis of new approaches to use crystallinity to enhance the resolution of membrane protein structures $[12,13]$. 
OmpF is originates from the outer membrane of Escherichia coli bacteria [14] which have a very asymmetric lipid bilayer comprising phospholipids on the inside and lipopolysaccharide (LPS) on the outer [12] (Fig1). LPS protects the cells from the external environment and consists of a sugar headgroup attached to a lipid tail. It is known that OmpF interacts strongly with LPS and we wished to recreate this condition in the minimal amphipol solubilized samples. The effect was striking since the addition of LPS caused the linear arrays to break down resulting in the formation of two dimensional crystals of OmpF (Fig. 1). This confirms that minimal amphipol is a "gentle" solubilizing system which does not inhibit weak interactions between membrane proteins. It suggests that reconstitution of weakly associated membrane complexes may be achieved by careful control of free Apol levels [15].
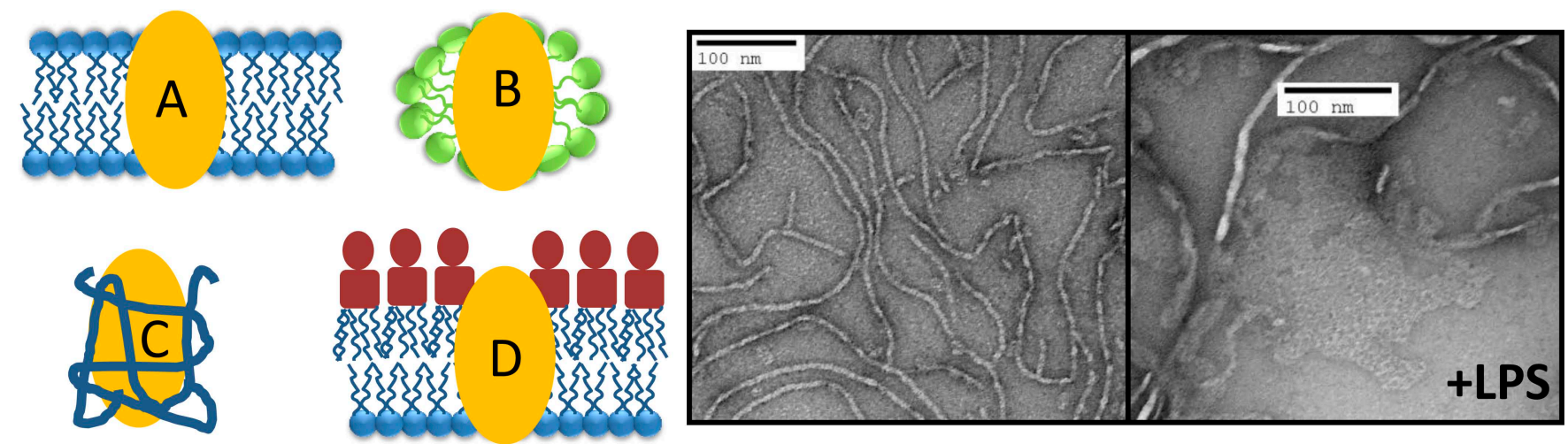

Figure 1. Left; cartoons of proteins in (A) lipid bilayer, (B) detergent micelles,(C)Amphipol, (D) E coli outer membrane with LPS shown in red. Right Negative stained EM comparison of OMPF protein in minimal APol (filaments) and with added LPS where the linear filaments break down to form 2D crystals.

1. R. Henderson and P. N. T. Unwin, Nature, 1975, 257, 28-32.

2. C. Tribet, et al., Proceedings of the National Academy of Sciences of the United States of America, 1996, 93, 15047-15050.

3. J. L. Popot, et al., Cellular and Molecular Life Sciences, 2003, 60, 1559-1574.

4. C. Tribet, et al., Biochimie, 1998, 80, 475-482.

5. M. Flötenmeyer, et al., Journal of Microscopy, 2007, 227, 229-235.

6. Y. Gohon, et al., Biophysical Journal, 2008, 94, 3523-3537.

7. T. Althoff, et al., Embo Journal, 2011, 30, 4652-4664.

8. T. L. Cvetkov, et al., Journal of Biological Chemistry, 2011, 286, 38168-38176.

9. E. Cao, et al., Nature, 2013, 504, 113.

10. M. Liao, et al., Nature, 2013, 504, 107.

11. J. R. Harris, Negative Staining and Cryoelectron Microscopy, Bios Scientific Publishers Ltd, Oxford, UK, 1997.

12. T. G. Baboolal, et al., Structure, 2008, 16, 371-379.

13. L. A. Clifton, et al., Journal of Biological Chemistry, 2012, 287, 337-346.

14. J. H. Lakey, et al., Biochimica Et Biophysica Acta, 1985, 817, 208-216.

15. WA thanks the Royal Thai Government for a Scholarship. We thank Helen Ridley for technical assistance. 